\title{
FIRE DETECTION ALGORITHMS USED BY A HETEROGENEOUS MULTI-AGENT SYSTEM
}

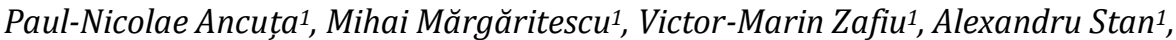 \\ Andrei Cristian Dinu ${ }^{1}$, Eduard Valentin Canale ${ }^{1}$ \\ ${ }^{1}$ National Institute of Research and Development in Mechatronics and Measurement Technique (INCDMTM), \\ 6-8 Pantelimon Road, Bucharest, Romania \\ Emails: paul.ancuta@incdmtm.ro,mihai.margaritescu@gmail.com,victor.zaf@gmail.com, \\ alexandrustan71.sa@gmail.com, andrei.dinu931@gmail.com, eduard.canale@gmail.com
}

\begin{abstract}
The SICOLET project proposes a heterogeneous collaborative multi-agent system consisting of a terrestrial vehicle equipped with a serial manipulator, a drone-type vehicle and an ekranoplan capable of semi-autonomous operation is determined by intelligent decision and control algorithms, designed for inspection and intervention in areas affected by various natural or anthropic disasters. Each agent is equipped with cameras, so the main data acquired by the system consist in digital images. This paper focuses on the fire detection algorithms, the fire being considered an important indicator associated with a disaster. Two image algorithms are presented. They are developed using NI Vision Assistant, a software tool for rapid prototyping and testing image processing applications. Besides software implementation in C language - which NI Vision Assistant generates by default - the paper presents a Python implementation of the first algorithm due to the fact that Python software is widely used on IoT devices.
\end{abstract}

Keywords: Image processing, Fire Detection, Image Segmentation, Multi-Agent System, Heterogeneous System.

\section{Introduction}

The consequences of disasters such as earthquakes, volcanic eruptions, hurricanes, floods or fires are particularly severe, manifesting themselves in loss of life and significant material damage. Disasters affect both densely populated urban and non-urban areas with varied relief. Although costly efforts are made to train intervention teams, due to difficult conditions in the field in the first hours or days after the event, rescue activities are difficult and often endanger the lives of rescuers. There are currently various remote-controlled terrestrial or aerial robotic systems that facilitate inspection and intervention in hard-to-reach affected areas. Due to the extremely diverse conditions, the mentioned systems can be effective in some cases, but inappropriate in others. For this reason, it is intended to use a multi-agent system (MAS) consisting of three different vehicles, hereinafter referred to as inspection / intervention agents, hence the name of heterogeneous system. Through their different characteristics, the three agents can complement each other, significantly expanding the capabilities of the system. Moreover, as the main scientific objective of the project, the aim is for the system to operate autonomously on certain segments of the intervention process, the three agents collaborating in different degrees with each other, based on communication and decision algorithms.

The main information collected by the three agents is based on digital images transmitted by cameras mounted on each agent. This paper focuses on the fire detection algorithms, because fire is appreciated an important indicator of an undesired event.

\section{Short Description of MAS}

The SICOLET MAS is composed by a control centre and the next agents:

- A terrestrial drone, equipped with a serial robot capable of intervention in the environment - figure 1:

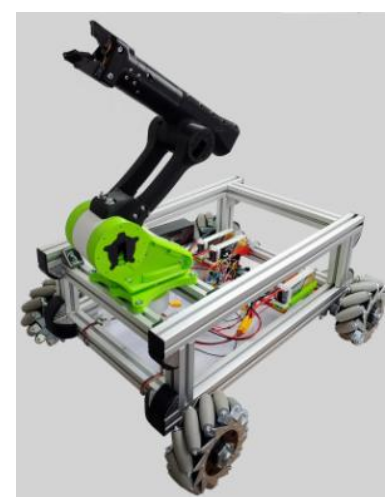

Figure 1: Agent 1 - the terrestrial drone 
- An aerial vehicle/ drone - figure 2:

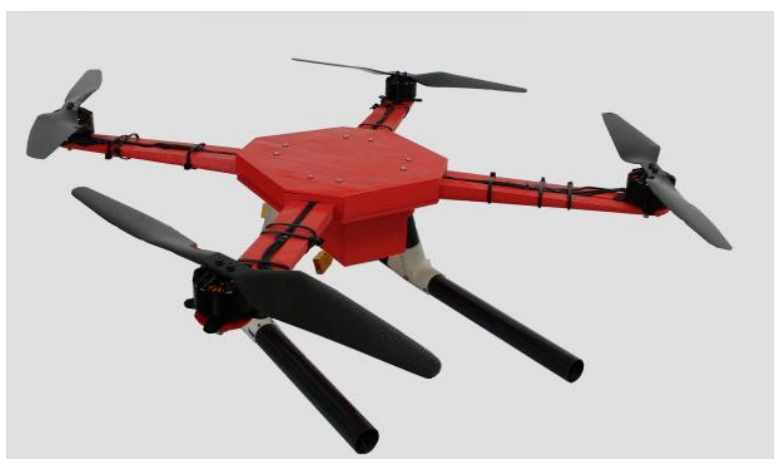

Figure 2: Agent 2 - the aerial vehicle

- An ekranoplan - figure 3 - is an autonomous lowaltitude flying vehicle based on the ground effect.

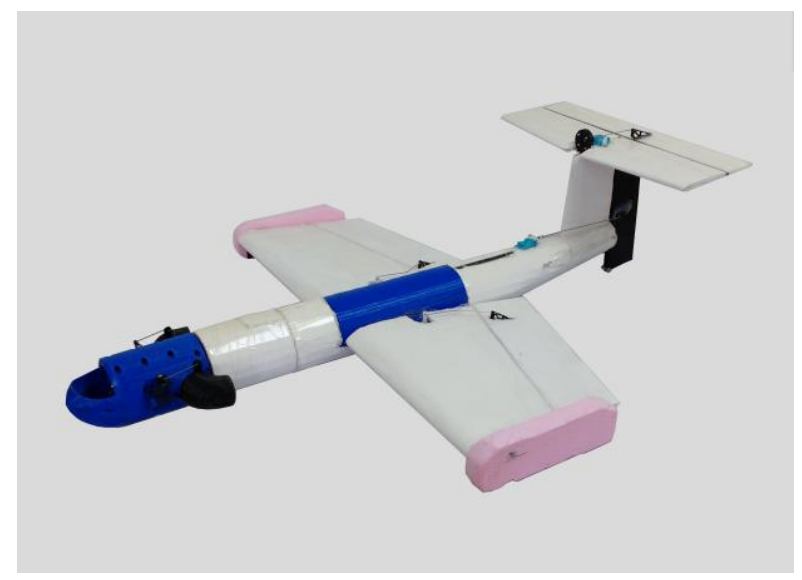

Figure 3: Agent 3 - the ekranoplan

All these agents are equipped with cameras, so the main data acquired by the MAS consist in digital images. The images can be processed either locally, inside each agent, or can be transmitted to the control centre.

Digital images are contained in dedicated graphical format files. A colour model describes how colours could be represented as tuple of numbers and may have associated one or more colour spaces. Some representative colour spaces are standard RGB, HSV, HSL. These colour spaces contain three distinctive channels. Each channel may be assimilated with a grey image. A histogram is a mathematical representation of the pixels' intensity distribution within a grey image

In our paper we used histogram-based image processing algorithms for fire detection. The idea is getting successive binary masks in order to filter the original image to obtain areas in the image that may contain fire representation. The processing pipeline extracts the most suitable colour planes (channel) from the original image's colour space and convert them into binary masks. by determining an optimal threshold value.

\section{Previous and Related Work}

Paper [1] presents an image-based real-time fire detection method using image processing on RGB colour model and CIE L*a*b colour channels. Paper [2] presents algorithms for fire detection using, RGB and $\mathrm{YCbCr}$ colour models. Papers [3] and [4] present algorithms for fire detection using RGB colour model. Paper [5] presents a method for fire detection in images using K-medoids clustering and Particle Swarm Optimisation procedures to better detect false alarm rate. Paper [6] presents a method to efficiently detect the flame in multiple scenes in an image. It uses a set of parametric representation named as Gradient Features (GF), to learn the features of flame colour changes in the image. Paper [7] proposes a novel method to detect fire and/or flames in real-time by processing the video data generated by an ordinary camera monitoring. It uses 1-D temporal wavelet transform and colour variation in fire-coloured moving regions using 2-D spatial wavelet. Papers [8] and [9] present a model for fire and smoke identification using Fuzzy Logic based image processing. Papers [10] and [11] present realtime video fire flame and smoke detection method.

Paper [12] presents several threshold methods that maybe applied to digital images in order to obtain a binary image.

\section{Material and Methods}

NI Vision Development module contains hundreds of image processing algorithms including filters. morphology, pattern matching, classification and many more. Threshold methods are described in [13].

The probability of occurrence of the grey level i is

$$
p(i)=\frac{h(i)}{\sum_{i=0}^{N-1} h(i)}
$$

where $\mathrm{h}(\mathrm{i})$ denotes the number of pixels in the image at each grey level value and $\mathrm{N}=256$.

Inter Variance method uses the probability of grey levels to determine the optimal threshold value $k$ for which the following expression is maximized:

$$
\sigma_{B}^{2}(k)=\frac{\left[\mu_{T} \omega(k)-\mu(k)\right]^{2}}{\omega(k)(1-\omega(k))}
$$

where:

$$
\begin{aligned}
& \mu(k)=\sum_{i=0}^{k} i p(i) \\
& \mu_{T}=\sum_{i=0}^{N-1} i p(i) \\
& \omega(k)=\sum_{i=0}^{k} p(i)
\end{aligned}
$$

Observation: Inter Variance method is an implementation of well-known Otsu algorithm for global automatic threshold of a histogram. 
Clustering method for automatic global threshold of a grey image involves obtaining a $\mathrm{k}$ value so that the condition.

$$
\frac{\mu_{1}+\mu_{2}}{2}=k
$$

is true, where $\mu_{1}$ is the mean of all pixels values from 0 to $\mathrm{k}$, and $\mu_{2}$ is the mean of all pixels values between $\mathrm{k}+1$ and 255 .

We will describe two algorithms for image processing.

The first algorithm uses RGB colour model of the analysed image. We used NI Vision software tool [13] which can be used for prototyping and testing image processing applications. The algorithm is recorded in a script file, which contains the processing functions and relevant parameters. The pipeline processing is presented in Figure 4.

The image processing steps are as follows:

1.Load original image from file

2.Save image in memory buffer for later use

3.Extract blue plane, which is a grey image

4.Apply a global automatic threshold in order to obtain a binary image

5.Reverse the binary image obtained in step\#4 in order to obtain Mask \#1

6.Save Mask\#1 in an image file

7.Retrieve the original image from memory buffer

8.Mask the original image with Mask\#1, obtaining a new colour image named "image1"

9.Extract the red plane from "image1"

10.Analyze logarithmic histogram

11.Apply an approximate global threshold in order to obtain Mask\#2

12.Dilate Mask\#2 image

13.Eliminate inner holes in Mask\#2 image

14. Save Mask\#2 in an image file

15. Mask "image1" with Mask\#2 in order to obtain the areas where fire exists

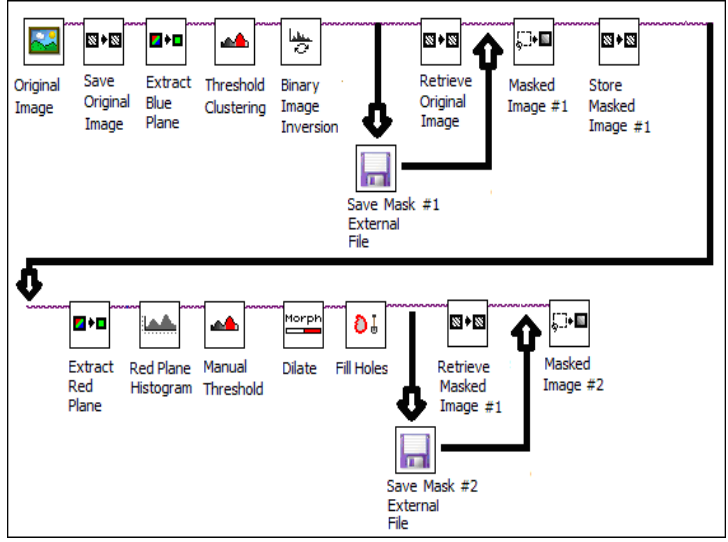

Figure 4: NI Vision script using RGB colour model

The NI Vision script described above can be automatically converted into a $\mathrm{C}$-code piece of software. However, we tried to achieve the following goals:
1.Eliminate the arbitrary user selection of threshold value at step \#11

2.Code the algorithm in Python.

We applied a 1-D Gaussian Filter [14] to the logarithmic histogram of the red plane in order to "smooth" the histogram, followed by the numerical calculus of the first derivate and second derivate. We chose the threshold point as the local minima closest to 255 where first derivate is zero and second derivate is positive.

For automatic threshold detection at step \#4, we used pieces of software from [15], grouped in otsu.py file. The Python code is presented below.

from scipy.ndimage import gaussian_filter1d

from matplotlib import pyplot as plt

from otsu import otsu, running variance, stretch, unstretch

import math

import numpy as np

import cv2

minval $=0$

maxval $=255$

\section{\# STEP 1}

image_orig= cv2.imread('forest5_test.bmp')

\# STEP 2

image $=$ image_orig.copy()

\# STEP 3

blue_plane= image[:,:,0]

\section{\# STEP 4}

image_grayblue=stretch(blue_plane)

print 0

threshold_otsu=int(otsu(image_grayblue)*maxval)

print(otsu(image_grayblue))

print(threshold_otsu)

print 0

,threshOtsu=cv2.threshold(blue_plane, threshold_otsu, 255, cv2.THRESH_BINARY)

contours1, hierarchy1 = cv2.findContours ( threshOtsu, cv2.RETR_TREE,

cv2.CHAIN_APPROX_SIMPLE)

for cnt1 in contours1:

cv2.drawContours(threshOtsu,[cnt1],0,255,-1)

\# STEP 5

mask $=$ cv2.bitwise_not(threshOtsu $)$

\# STEP6

cv2.imwrite('Mask1.bmp', mask)

\# STEP 7

mask1=cv2.imread('Mask1.bmp', cv2.IMREAD_UNCHANGED )

\# STEP 8

image $1=$ cv2.bitwise_and(image,image, mask=mask1) 
\#STEP 9

red_plane = image $1[:,:, 2]$

\section{\# STEP 10}

histo_red = cv2.calcHist([red_plane], [0], None, [256], $[0,256])$

log_histo_red

=np.log(1+histo_red.astype(np.float))

\#adjust extreme values of the histogram

log_histo_red[0] = log_histo_red[1]

log_histo_red[255]=log_histo_red[254]

print(log_histo_red)

$\operatorname{mMax}=\max (\log$ histo_red $)$

arr_log_histo_red = np.array(log_histo_red)

\# smooth

smooth = gaussian_filter1d(arr_log_histo_red.T[0], 3)

\#compute first derivate

smooth_d1 $=$ np.gradient (smooth)

zeroD1= np.where $($ np.diff(np.sign( smooth_d1) $)$ [0]

\# compute second derivative

smooth_d2 = np.gradient(np.gradient(smooth))

zeroD2=np.where $($ np.diff( np.sign $($ smooth_d2) $))[0]$

histo_red[0]=histo_red[1]

histo_red[255]=histo_red[254]

plt.plot(histo_red, label='Original Histogram')

plt.legend(bbox_to_anchor $=(0.5,0.5))$

plt.show 0

plt.plot(arr_log_histo_red,

label='Logarithmic histogram')

plt.plot(smooth,

label='Smoothed Histogram')

plt.plot(smooth_d1/ np.max(smooth_d1),

label= 'First Derivate')

plt.plot(smooth_d2 / np.max(smooth_d2),

label= 'Second Derivate')

plt.axhline $(y=0$, color=' $k$ ')

\section{\# STEP 11}

last_local_minima $=0$

for $i, x z e r o$ in enumerate(zeroD1, 1):

if smooth_d2 [xzero] $>0$ :

plt.axvline( $\mathrm{x}=\mathrm{xzero}$, color=' $\mathrm{k}$ ')

last_local_minima=xzero

plt.legend(bbox_to_anchor $=(0.5,0.5))$

plt.show 0

threshold_redplane=last_local_minima

,mask=cv2.threshold(red_plane, threshold_redplane, 255 , cv2.THRESH_BINARY)

\section{\# STEP 12}

dilation_size $=1$

element $=$ cv2.getStructuringElement (

cv2.MORPH_RECT,(2*dilation_size+1,

$2 *$ dilation_size+1), (dilation_size, dilation_size))

dilation_mask2 = cv2.dilate(mask, element)

\section{\# STEP 13}

contours2, hierarchy2 = cv2.findContours(mask, cv2.RETR_TREE, cv2.CHAIN_APPROX_SIMPLE)

for cnt2 in contours2:

cv2.drawContours(mask, [cnt2],0,255,-1)

\section{\# STEP 15}

cv2.imwrite('Mask2.bmp', mask)

\section{\# STEP 15}

mask2=cv2.imread('Mask2.bmp', cv2.IMREAD_UNCHANGED)

image $2=$ cv2.bitwise_and(image 1, mask=mask2)

\#Display images

cv2.imshow("Initial",image)

cv2.imshow ("Otsu",threshOtsu)

cv2.imshow("Mask1",mask1)

cv2.imshow ("Image 1",image1)

cv2.imshow ("Mask2",mask2)

cv2.imshow("Image 2",image2)

cv2.waitKey(0)

cv2.destroyAllWindows()

The second algorithm uses HSV/HSL colour models of the analysed image is presented in Figure 5.

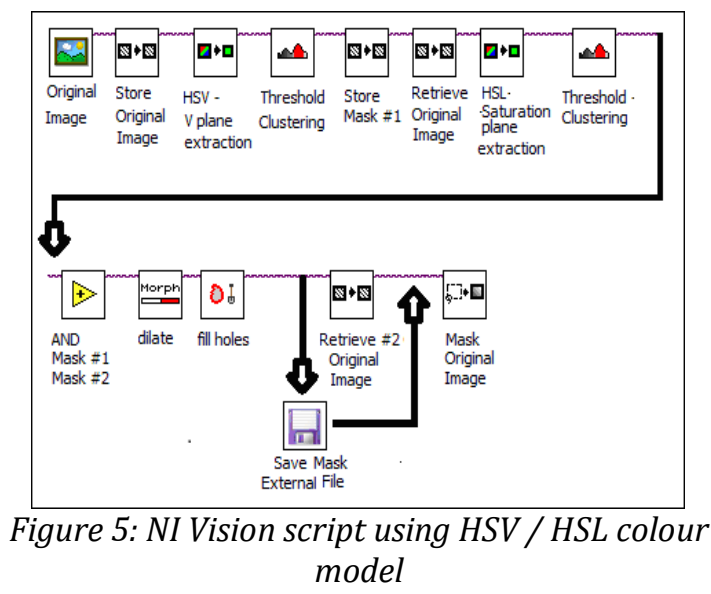

The image processing steps are as follows:

1.Load original image from file

2.Save image in memory buffer for later use

3.Extract $\mathrm{V}$ plane, which is a grey image

4.Apply a global automatic threshold in order to

obtain a binary image as Mask\#1

5.Store Mask \#1

6. Retrieve original image from memory buffer

7.Extract Saturation plane from image

8.Apply a global automatic threshold

9.Apply AND operator on Mask\#1 and current image

in order to obtain Mask\#2

10.Dilate Mask\#2 image

11.Eliminate inner holes in Mask\#2 image

12.Save Mask\#2 in an image file

13. Mask original with Mask\#2 in order to obtain the areas where fire exists 


\section{Experimental Results}

The picture used as input data for the first algorithm contains a large area of smoke (upper-left) and an area of sky (upper-right). These areas may be detected as false positive fire areas. They are eliminated using a binary mask image obtained by applying a threshold to the image's blue plane. The representative images are depicted in Figure 6 to Figure 10 and also in Figure 13.

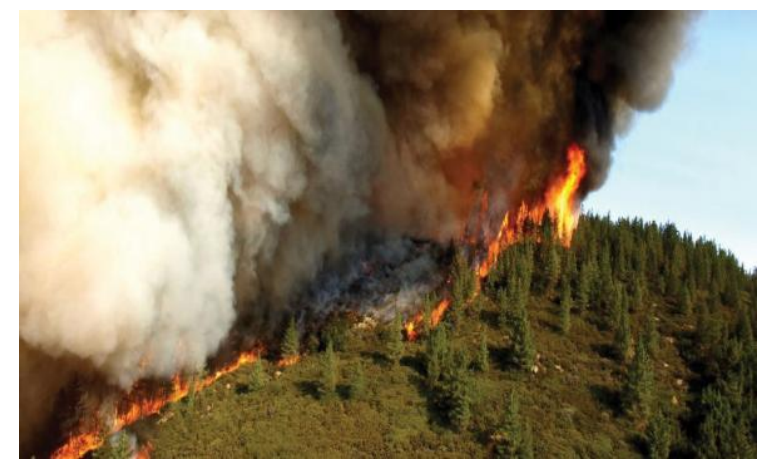

Figure 6: Original Image for the first algorithm

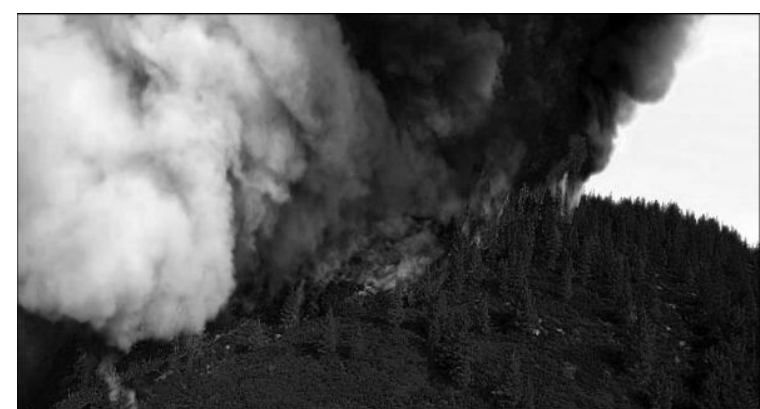

Figure 7: Blue Plane of the Original Image

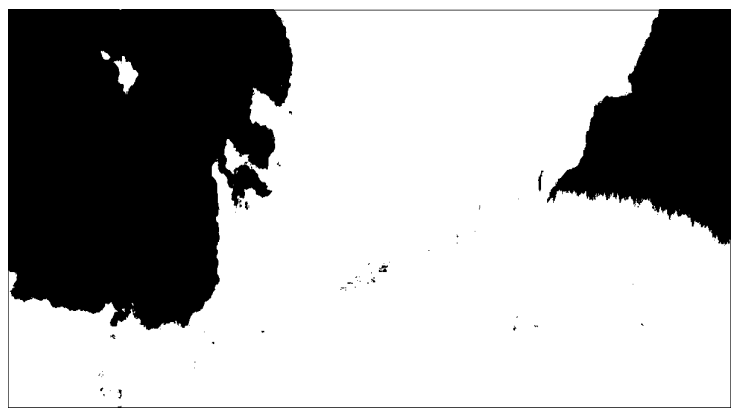

Figure 8: Mask \#1

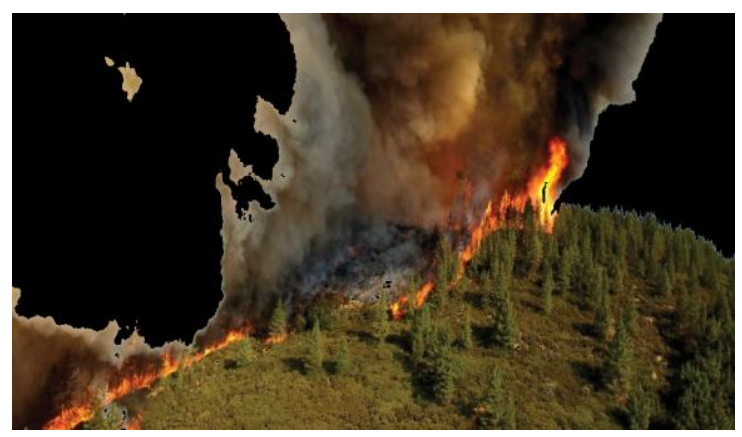

Figure 9: Original image masked (Image 1)

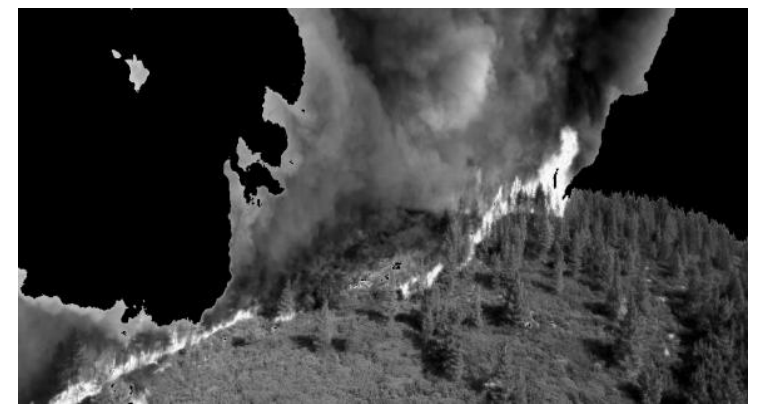

Figure 10: Red Plane of Image 1

Figure 11 presents the logarithmic representation of histogram of the red plane, generated by NI Vision Assistant script.

Arbitrary threshold value is represented with an arrow.

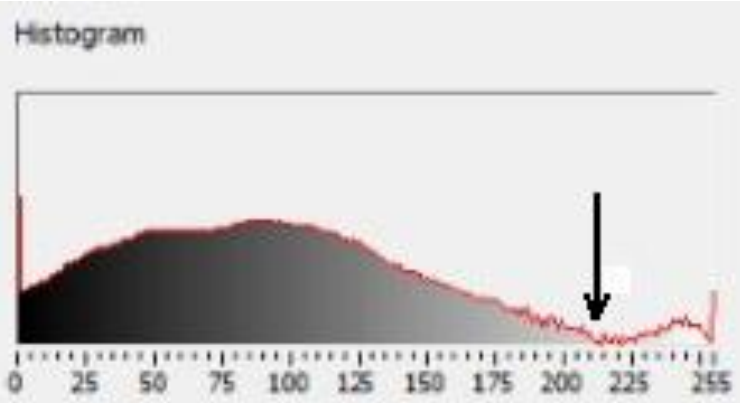

Figure11: NI Vision Assistant manual threshold

Figure 12 presents the automatic threshold detection based on first and second derivate of the smoothed logarithmic histogram.

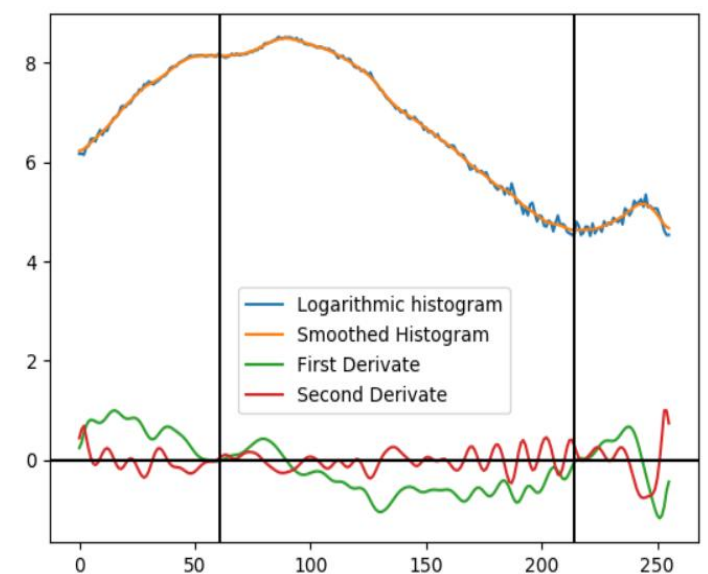

Figure 12: Threshold obtained using Python code

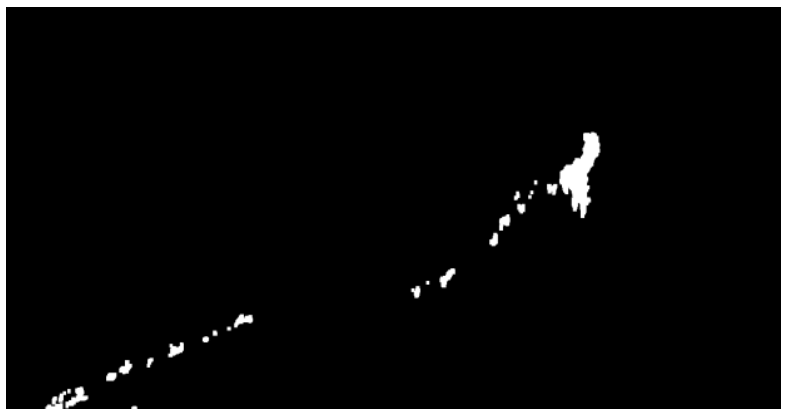

Figure 13: Mask \#2 
Figure 14 presents fire detection carried out by successive binary masks applied to the original image.

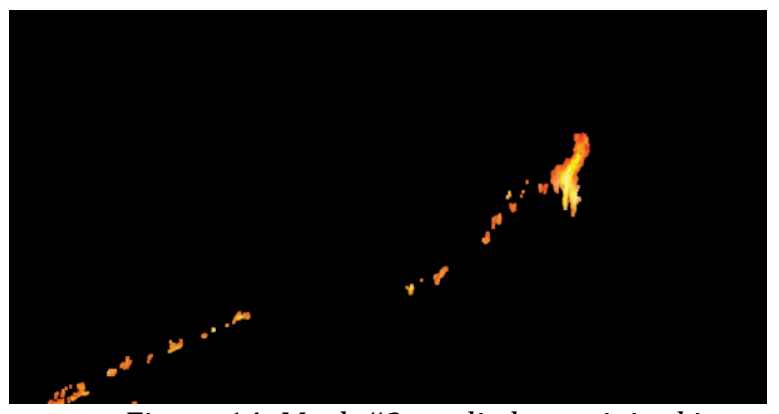

Figure 14: Mask \#2 applied on original image

The input data for the second algorithm is a picture from a city fire action.

The threshold values for the two mask2 are automatically detected. The representative images are depicted in Figure 15 to Figure 21.

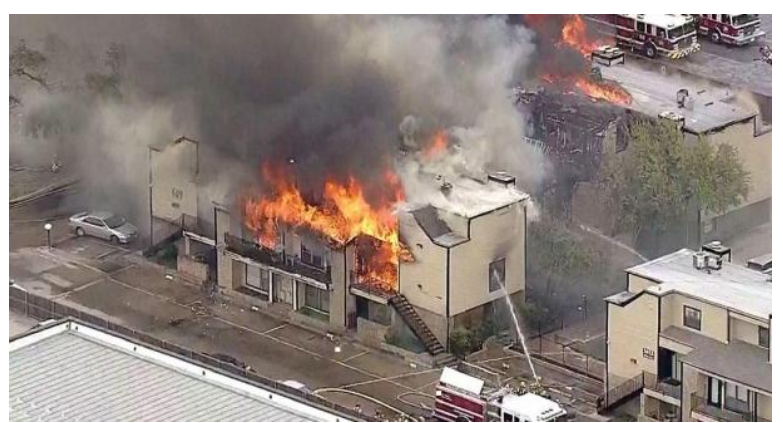

Figure 15: Original Image for the second algorithm

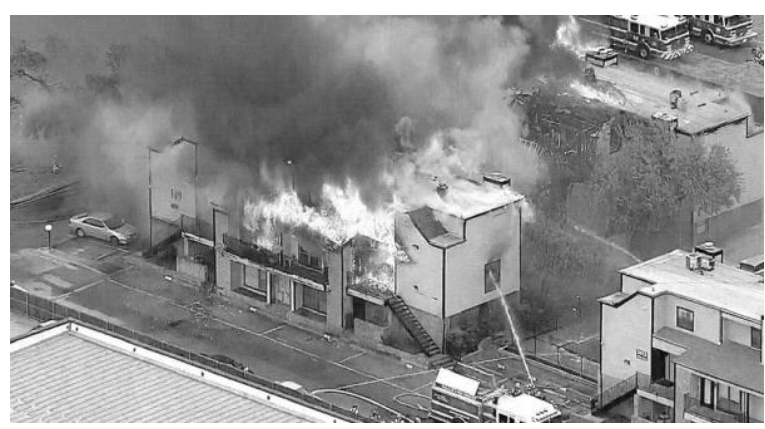

Figure 16: V plane from HSV colour space

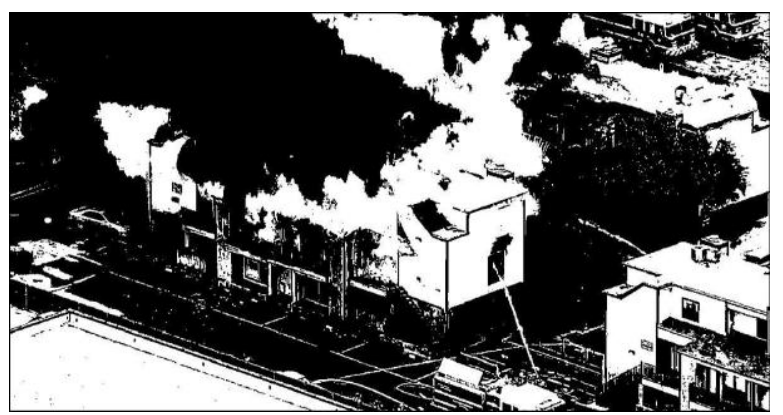

Figure 17: Mask \#1

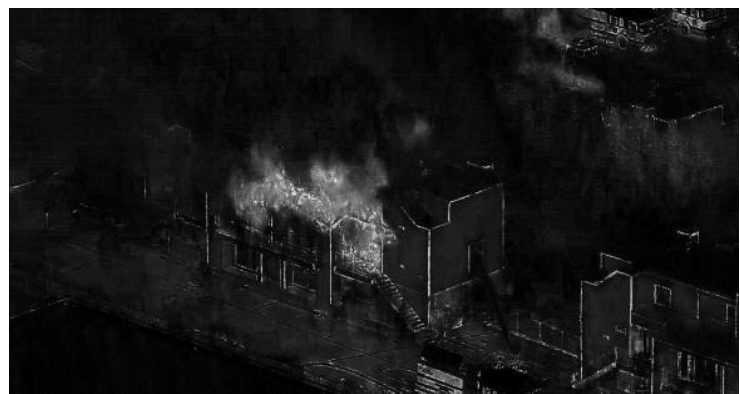

Figure 18: H plane from HSL colour space

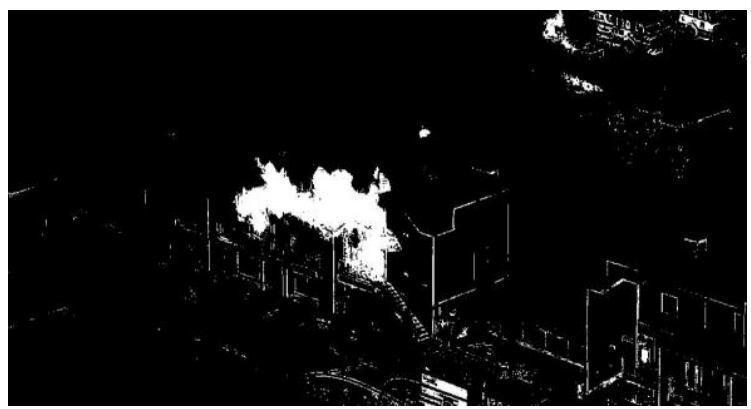

Figure 19: Mask \#2

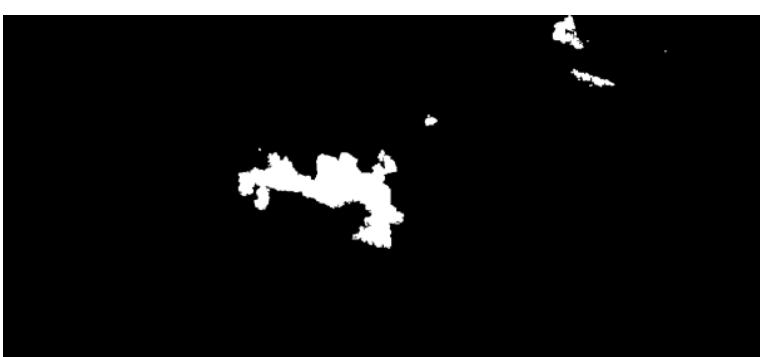

Figure 20: Mask\#2 after morphology operations

Figure 21 presents fire detection carried out by successive binary masks applied to the original image.

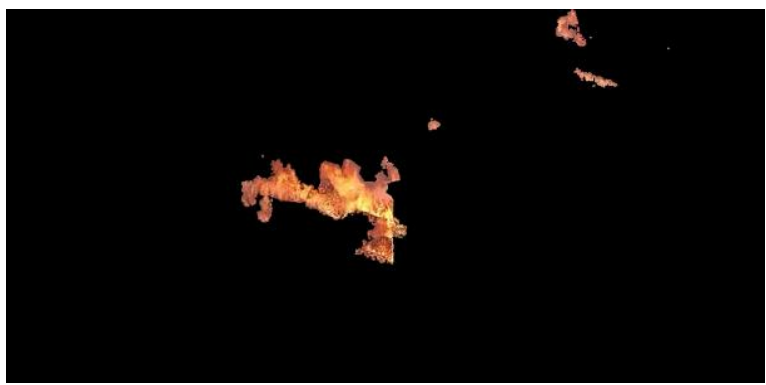

Figure 21: Fire detection in original image

\section{Conclusions}

Fire detection in digital images is a challenging task. The presence in image of clear sky, smoke and clouds may produce false positive results. Image processing algorithms that are describes in the paper use histogram analyse of the proper colour planes extracted from initial images. 
The colour planes are turned into binary masks by applying a threshold method. Different image processing scenarios are developed with NI Vision Assistant, a powerful software tool. Paper presents also a Python implementation of fire detection in image, which is suitable for IoT devices. Future works will be focused on using Machine Learning and Deep Neural Networks for fire detection in image processing.

\section{Acknowledgements}

This work was supported by a grant of the Romanian Ministry of Education and Research, Research program NUCLEU, contract no. 17N/2019, project number PN 19.24.01.01 - SICOLET.

\section{References}

[1] Turgay Celic, Fast and Efficient Method for Fire Detection Using Image Precessing, ETRI Journal, Volume 32, Number 6, December 2010, pp881890

[2] Supriya Sameer Nalawade, Fire Detection System using RGB Color Model, International Journal of Engineering Science and Computing, May 2018, Volume 8, Issue \#5, pp 17516...17518

[3] Jing Shao, Guanxiang Wang, Wei Guo,An ImageBased Fire Detection Method Using Color Analysis, 2012 International Conference on Computer Science and Information Processing, pp 419-422

[4] Ping-He Huang, Jing-Yong Su, Zhe-Ming Lu, JengShyang Pan, A Fire-Alarming Method Based on Video Processing, Proceedings of the 2006 International Conference on Intelligent, Information Hiding and Multimedia Signal Processing (IIH-MSP'06), pp. 359-364

[5] Oluwarotimi Giwa, Abdsamad Benkrid, Fire detection in a still image using color information, https://www.researchgate.net/publication/3237 23024_Fire_detection_in_a_still_image_using_colo r_information, accessed at 10.09 .2020
[6] Zhu Liping, Li Hongqi, Wang Fenghui, Lv Jie, Sikandar Ali, Zhang Hong, A Flame Detection Method Based on Novel Gradient Features, Intell. Syst. 2020; 29(1): 773-786, https://doi.org/10.1515/iisys-2017-0562

[7] B. Uğur, Töreyin, Yiğithan Dedeoğlu, Uğur Güdükbay, A. Enis Cetin, Computer vision based method for real-time fire and flame detection, Pattern Recognition Letters 27 (2006) 49-58

[8] Sushil Garg, Balaji R. Sharma1, Kelly Cohen, Manish Kumar, A Fuzzy Logic Based Image Processing Method for Automated Fire and Smoke Detection, https://www.researchgate.net/publication/2583 40149, accessed at 25.09.2020

[9] Turgay Çelik, Hüseyin Özkaramanlı, Hasan Demirel, Fire and smoke detection without sensors: image processing based approach, 15th European Signal Processing Conference (EUSIPCO 2007), Poznan, Poland, September 3-7, 2007, pp 1794-1798

[10] Chunyu Yu, Zhibin Mei, Xi Zhang, A Real-time Video Fire Flame and Smoke Detection Algorithm, Procedia Engineering, Volume 62, 2013, Pages 891-898, https://www.sciencedirect.com/science/article/ pii/S1877705813013222,accessed at 9.09.2020

[11] Margarita Favorskaya, Anna Pyataeva, Aleksei Popov, Verification of Smoke Detection in Video Sequences Based on Spatio - temporal Local Binary Patterns, Procedia Computer Science, Volume 60, 2015,pp.671-680, https://www.sciencedirect.com/science/article/ pii/S1877050915023327,accessed at 9.09.2020

[12] Mehmet Sezgin, Bülent Sankur, Survey over image thresholding techniques and quantitative performance evaluation. Journal of Electronic Imaging 13(1), 2004, pp. 146-165

[13] http://zone.ni.com/reference/en-XX/help/ 370281AG-01/nivisionconcepts/thresholding/ accessed at 28.09.2020

[14] http://www.ni.com/pdf/manuals/372228m.pd f accessed at 29.09.2020

[15] https://docs.scipy.org/doc/scipy/reference/ge nerated/scipy.ndimage.gaussian_filter1d.html accessed at 29.09.2020

[16] github.com/CellProfiler/centrosome/tree/mast er/centrosome accessed at 29.09.2020 